\title{
CONTROL DESIGN AND ANALYSIS OF CRUISE CONTROL SYSTEM
}

\author{
$\mathbf{1}_{\text {Victoria Oguntosin and }}{ }^{\mathbf{2}}$ Jamiu Olasina \\ ${ }^{1,2}$ Department of Electrical \& Information Engineering, \\ Covenant University, Ota, Ogun State. \\ ${ }^{2}$ Department of Computer Engineering, Federal Polytechnic, \\ Ilaro, Ogun State. \\ Email: $\left\{{ }^{1}\right.$ victoria.oguntosin@ covenantuniversity.edu.ng, \\ 2jamiuolasina@gmail.com $\}$
}

\begin{abstract}
This paper is aimed at modeling a cruise control system and examining its characteristics based on its root locus and frequency domain control strategies. This system is actualized with the aid of MATLAB for simulation and control purposes. The specifications of steady state error less than $1 \%$, overshoot less than $5 \%$ and rise time less than 1.5 seconds were achieved using the developed model.
\end{abstract}

Key Words - cruise control, control design.

\section{Introduction}

A system is said to be a cruise control system if it has technical skill to maintain a certain set speed for a particular length of time in as much there won't be any human intervention. This phenomenon would be studied in a car as a system. Also, this tremendous technology is very important at highway where a constant speed is to be maintained, and as such will reduce driving fatigue and as the case may be, the driver won't need to tamper with any car components as at when the cruise system is activated. In real life, cruise control can be activated when a desired speed is reached, and immediately the driver presses the ON button on the steering mounted controls, the system is automatically deactivated due to interruption from the driver [1]. Cruise control system has a lot of advantages and some of these are: Comfort diving as the driver does not need to press accelerator during long journey; the system makes fuel consumption to be more economical and the driving fatigue equally reduced a great deal. Although, the system is not good when there is heavy traffic on the road. This is so because; the driver cannot keep the vehicle at constant speed for long when there is heavy traffic [2]. Cruise control is applied to several automatic cars such as Mitsubishi [3] to control the speed. [4] proposed in their studies, feed forward controller that take into serious consideration, elimination of gravitational and wind disturbances effect. This system was implemented with AMR microcontroller based robotic application and C++ programing language. The use of GPS was later proposed not to control the speed alone but to operate the vehicle in safe speed mode. The GPS monitored the state of the vehicle speed at normal. ARM Processor used to reduce the vehicle speed to normal state whenever it receives signal of abnormality from GPS [5], [6]. Presently, the state of the art according to [7], involves a cruise control system to predict future hazard and automatically adjust the system parameters, so that such accident will not occur. Evaluation was done for the model and the model detected changes in lane with $99.2 \%$ accuracy with an average error of $0.065 \mathrm{~m}$ achieved.

Advanced cruise control that is already in use includes adaptive cruise control, antilock braking, automatic parking, pedestrian protection system and blind spot detection. The contributions of designing a control system for cruise control was achieved by [8] which involved modeling the adaptive cruise control in Petri Nets, analyzing the functionality of Petri nets, verifying the adaptive cruise control process, and implementing controller and fault tolerance techniques [9]. Cruise control can also be employed to control the speed of robotic systems [10][11].

This work is aimed at the modeling and control design of a cruise control system to achieve a fixed set of design specifications such as steady state error, settling time, percentage overshoot.

\section{Methodology}

Automatic cruise control is achieved by measuring the speed of a car and also monitors the speed by comparing it to the reference speed no matter what the external disturbance is all about. At the same time the throttle is to be adjusted according to the established control law. From Figure 1, the mass of the car is represented by $\mathrm{m}$, which is acted upon by the force, pull$\mathrm{u}$ that is generated at the interface between the tire and the surface of the road. It is also assumed of the model that ' $u$ ' can be controlled while the dynamic of the power train is neglected. The oppositional pull, $\mathrm{R}$ is assumed to be varied linearly with velocity of the car and it acts opposite to the motion of the car.

Horizontal resolution of vectored forces and application of Newton's second law, the final model is given as: $\boldsymbol{m} \dot{\boldsymbol{v}}+\boldsymbol{b} \boldsymbol{v}=$ $\boldsymbol{u}$, and also, the output equation is assumed to be $\boldsymbol{y}=\boldsymbol{v}$ since, the action of interest is controlling the speed of the car. 


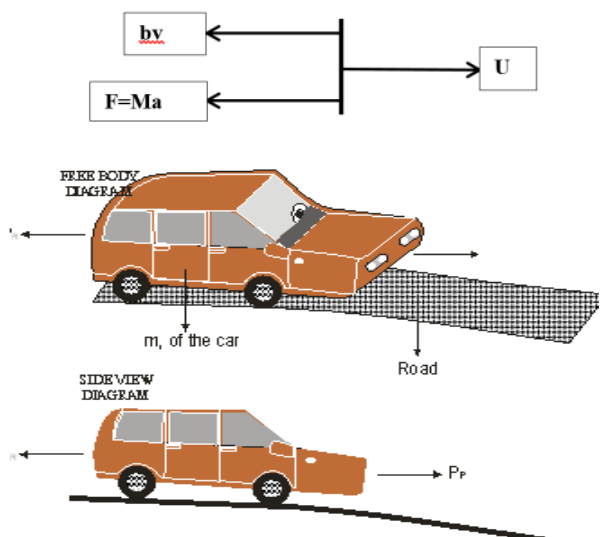

Fig. 1 Free Body Diagram and Side view diagram of a Car for Cruise Control.

Where $\mathrm{m}$ is the mass and $\mathrm{b}$ is the damping factor. Let these parameters of interest be, $m=1500 \mathrm{Kg}$ and $b=$ $75 \mathrm{~N} . \mathrm{s} / \mathrm{m}$. The state model for the system is therefore:

and

$$
\dot{v}=\left[-\frac{b}{m}\right][v]+\left[\frac{1}{m}\right][u]
$$

$$
y=[1][u]
$$

The model arrived at is according to the FBD broken down into the form below:

$$
\begin{aligned}
b v+F=u, b v+ & m a=u, b v+m \frac{d v}{d t}=u, b v+m \dot{v} \\
& =u
\end{aligned}
$$

Also, the transfer function for this system is derived below:

$$
\begin{gathered}
\begin{aligned}
G(s)=\frac{V(s)}{U(s)}=C & (s . I-A)^{-1} B+D, \text { where } A=\frac{-b}{m}, B \\
& =\frac{1}{m}, C=1, \quad(4)
\end{aligned} \\
G(s)=1 \times\left(s . I+\frac{b}{m}\right)^{-1}\left(\frac{1}{m}\right)+0=\left(s . I+\frac{b}{m}\right)^{-1}\left(\frac{1}{m}\right) \\
=\frac{\frac{1}{m}}{s+\frac{b}{m}}=\frac{1}{m s+b}
\end{gathered}
$$

$\mathrm{G}(\mathrm{S})=$ Transfer Function, $\mathrm{U}(\mathrm{S})=$ Input and $\mathrm{V}(\mathrm{S})=$ Output.

\section{Analysis of the model}

The beauty of any model is to be able to be mathematically analyzed. The deductions arrived at will then help to fully explain the nature of such system in real sense. Also, it will help to see the system responsiveness with respect to time and frequency. Besides, we can choose design parameters to amend the system for stability, speed of response, steady state error, and oscillatory- free system. The analysis of this system is based on the following parameters and specifications: steady state error is less than $1 \%$, overshoot is $<5 \%$ and rise time $<1.5$ seconds. The response of the system to a step input force of $750 \mathrm{~N}$ is first simulated and the graphical illustration is shown in Figure 2.

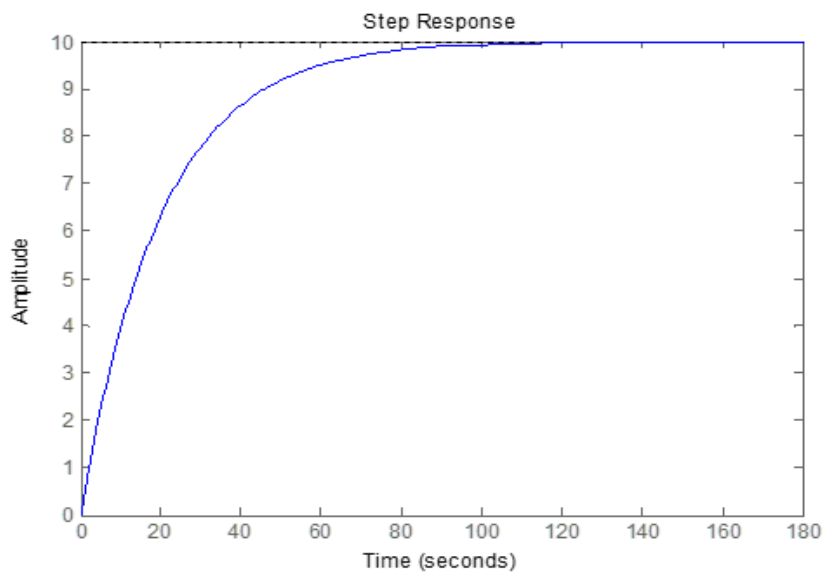

Fig. 2 Simulation of Open loop control system

The system reached its set value of $10 \mathrm{~m} / \mathrm{s}$ at no oscillation and no overshoot. Although the rise time is approximately 50s, this value is low, and it is required for a better closed-loop control system.

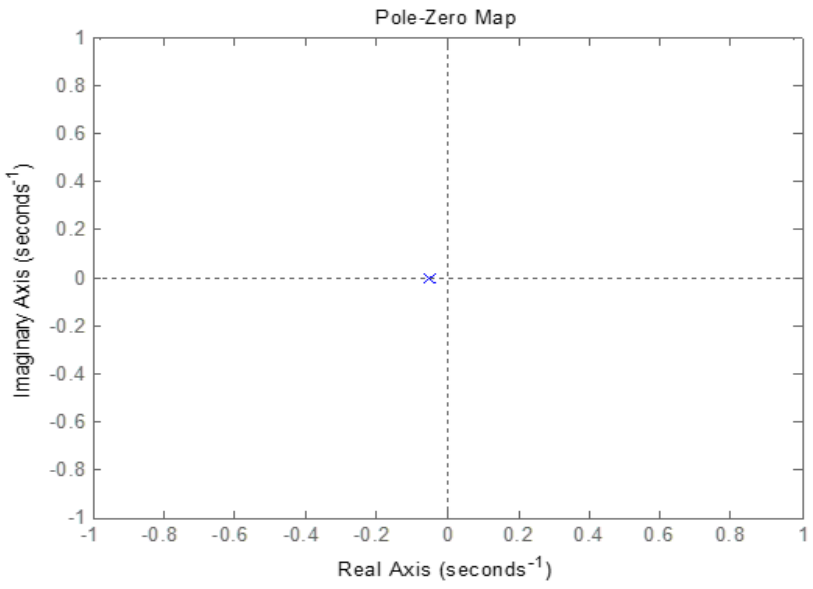

Fig. 3 The pole-zero plot of the system

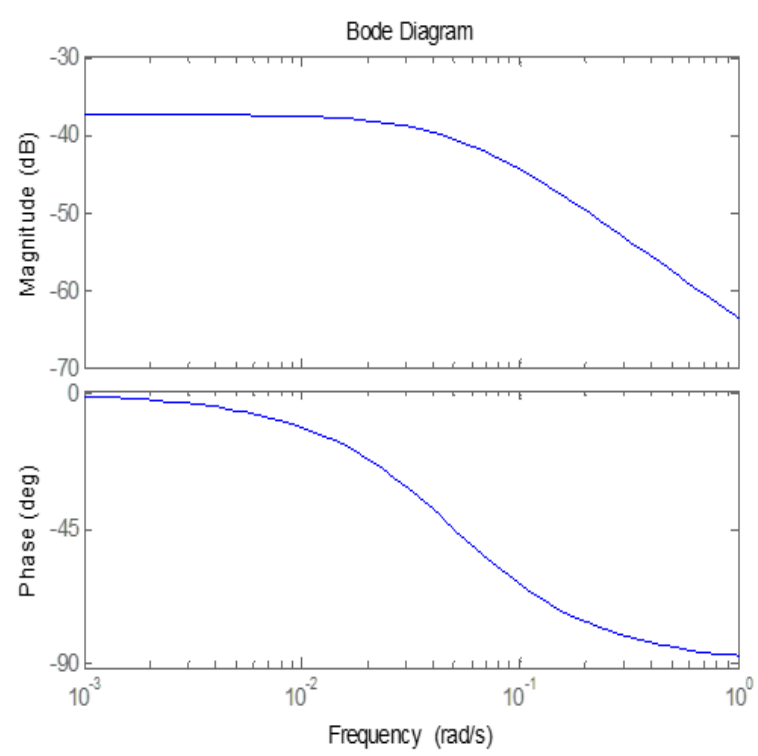

Fig. 4 The Bode plot of the system. 
The pole-zero plot of the equivalent open loop system in Figure 3 shows that there is no oscillation due to the fact that only a pole exists as real and negative. Now, a controller to meet the larger value of $\mathrm{b} / \mathrm{m}$ should be designed because, the greater the value of $\frac{b}{m}$, the faster the system becomes to reach the steady state.

As shown in Figure 4, the Bode plot exhibited a system property with $-61.6 \mathrm{~dB}$ of magnitude and $-87.1^{\circ}$ phase at the corner frequency of $0.803 \mathrm{rad} / \mathrm{s}$ and it roll-off at high frequencies of $0.992 \mathrm{rad} / \mathrm{s}$.

\subsection{PID Controller for the System}

Figure 5 is a typical PID closed loop representation of the system, and the consideration is given to the unity system. The PID of this system has a standardized mathematical equation:

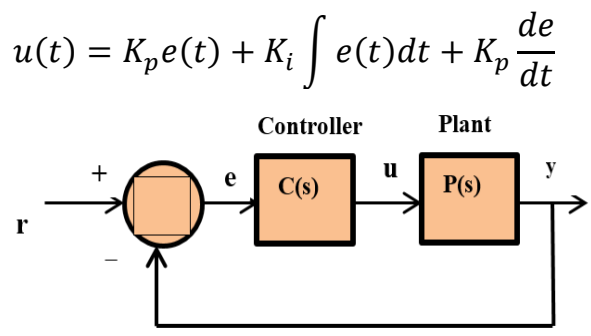

Fig. 5 The PID controller for the system

From Figure 5, the reference input and measured output are compared to generate the error (e) which is fed to the controller, at which the error is administrated through proportional parameter, also integrated and finally differentiated to generate control output $(u)$ that is later presented to be new input to the plant (or system or model). The final actual output generated is released to be further compared with the desire input. The process continues on and on like that.

The Laplace function of the PID equation above is manipulated through to generate the result shown below, when all initial conditions are assumed zero

$$
K_{p}+\frac{K_{i}}{s}+K_{d} s=\frac{K_{d} s^{2}+K_{p} s+K_{i}}{s}
$$

The PID controller will be able to do the task: the proportional part will reduce $t_{r}$ and $e_{s s}$, the integral part will eliminate the error but the transient response will slow down, and the derivative part will drive the system to stability, reduce the overshoot and improve the transient response.

\subsection{Root Locus Control Design of the System}

Figure 6 shows the root locus of the system.

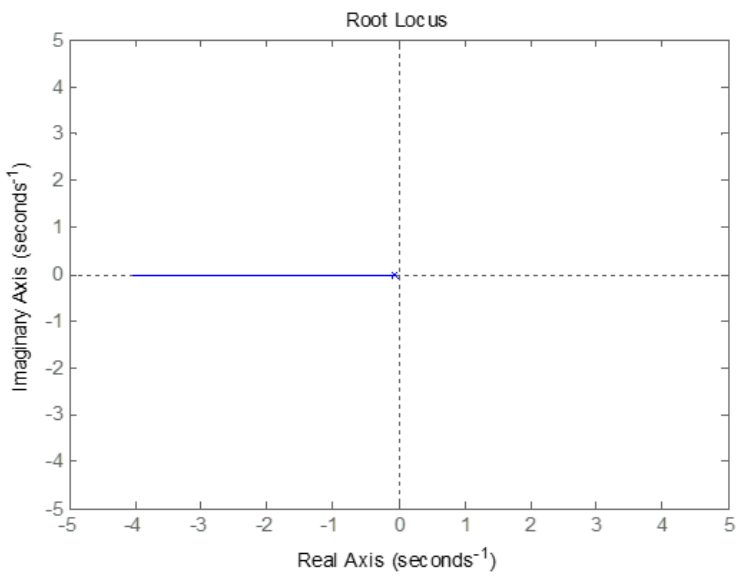

Fig. 6 Root locus plot of the system

If overshoot of $5 \%$ is required, a $\xi>0.7$ would be needed while the rise time of $1 \mathrm{~s}$ is possible; a natural frequency above $1.8 \mathrm{rad} / \mathrm{s}$ would be fine as shown in Figure 7. The desired poles on the locus is chosen, a P-only controller is needed for the poles to the be in the desired region. The value chosen for $\mathrm{K}$ is 2822.

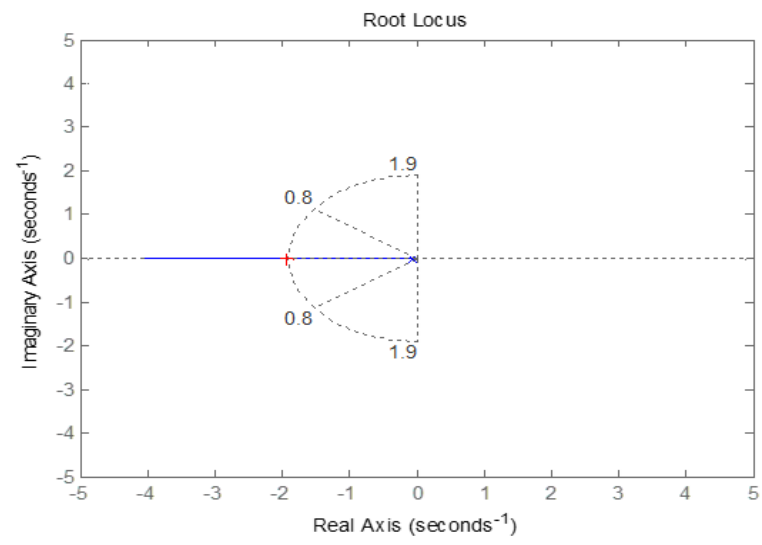

Fig. 7 Root locus plot of the system for a required overshoot of 5\%

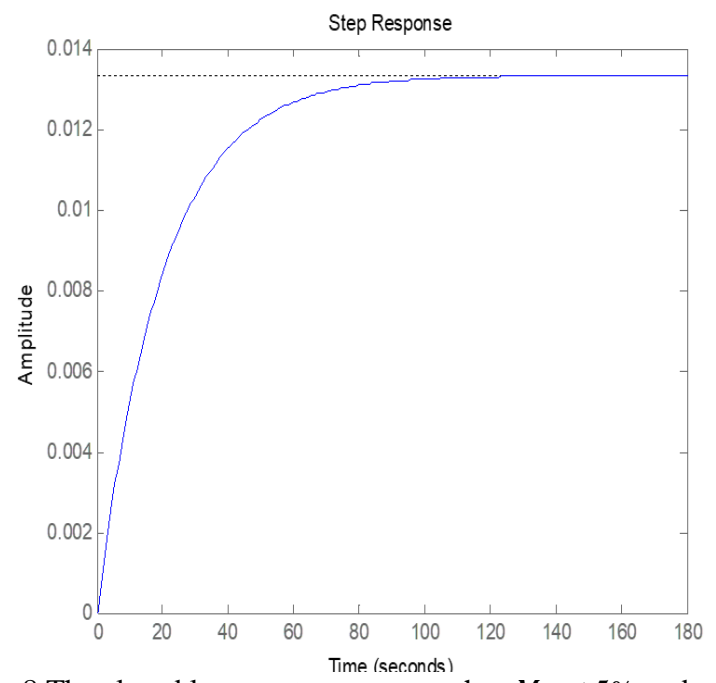

Fig. 8 The closed loop system response has $M_{p}<5 \%$ and a $t_{r}$ of $0.178 \mathrm{~s}$.

From Figure 8, the response has an overshoot $\left(M_{p}\right)<5 \%$ and 
a $t_{r}<1$ second ( 0.178 Seconds). The design specifications relating to the overshoot and rise time have been therefore achieved using root locus control.

\subsection{Frequency Domain for the System Design}

In this section, the system and parameter specifications are unchanged to carry out control design using frequency domain methods. A unity feedback system is equally considered. The specifications and design parameters are given as: step input force $=750 \mathrm{~N} ; \mathrm{m}=1500 \mathrm{Kg} ; \mathrm{b}=75 \mathrm{~N} . \mathrm{s} / \mathrm{m}$; desired speed of $10 \mathrm{~ms}^{-1}$. The performance specifications are given as: $t_{r}<5 \mathrm{sec} ; M_{p}<10 \%$; and $e_{s s}<2 \%$. The frequency response is examined by the use of open loop system. The transfer function for the system is:

$$
\frac{Y(S)}{E(S)}=\frac{K_{p}}{m s+b}, \text { where } K_{p}=1
$$

Also, the system must be stable for Bode plot to be examined with the system. For the system, the low frequency gain is $37.5 \mathrm{~dB}=0.00101$ which would be:

$$
\begin{aligned}
& \text { ss error }=\frac{1}{1+M_{\omega \rightarrow 0}} \times 100 \% \frac{1}{1+0.00101} \times 100 \%= \\
& 0.9989 \times 100 \%=99.89 \% \quad(8),
\end{aligned}
$$

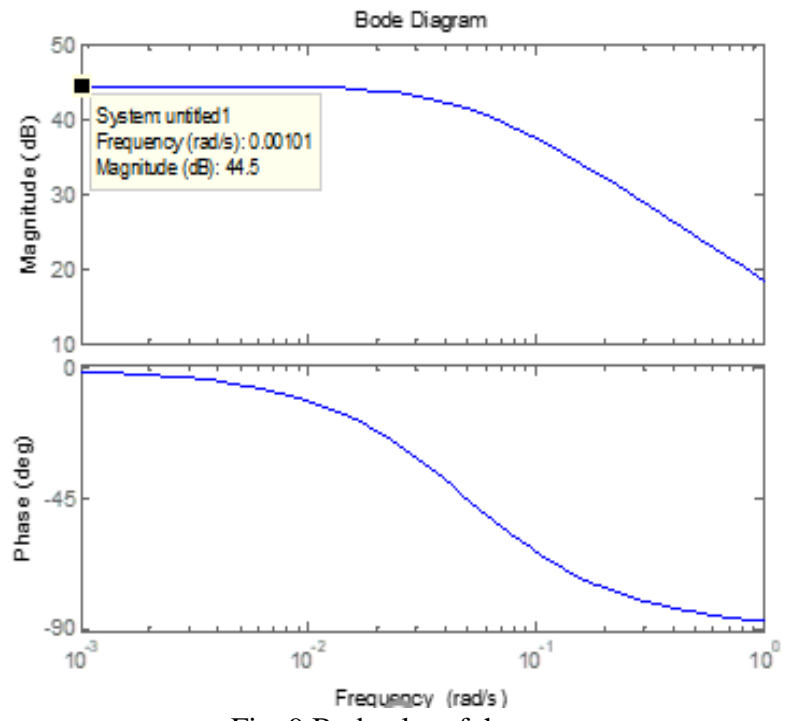

Fig. 9 Bode plot of the system

Low frequency gain needs to be increased, so that the steadystate error could still be improved. Specifically, the error is less than $1.2 \%$ approximately. But it is equally good if it is lesser than that. Let $1 /(1+\mathrm{M})=0.11$ and it implies $\mathrm{M}=8.0909$ $=17.98 \mathrm{~dB}$. Therefore, to reach the desired ss error using $\mathrm{P}$ controller only requires a $K_{p}>82.02 \mathrm{~dB}=12616.76=12617$ approximately. Figure 9 shows the Bode diagram of the compensated open-loop system.

From Figure 9, the low frequency magnitude is $44.5 \mathrm{~dB}$. The behavior of the step-response diagrammatically is shown in Figure 10. As shown, the system could be further improved by a lag compensator

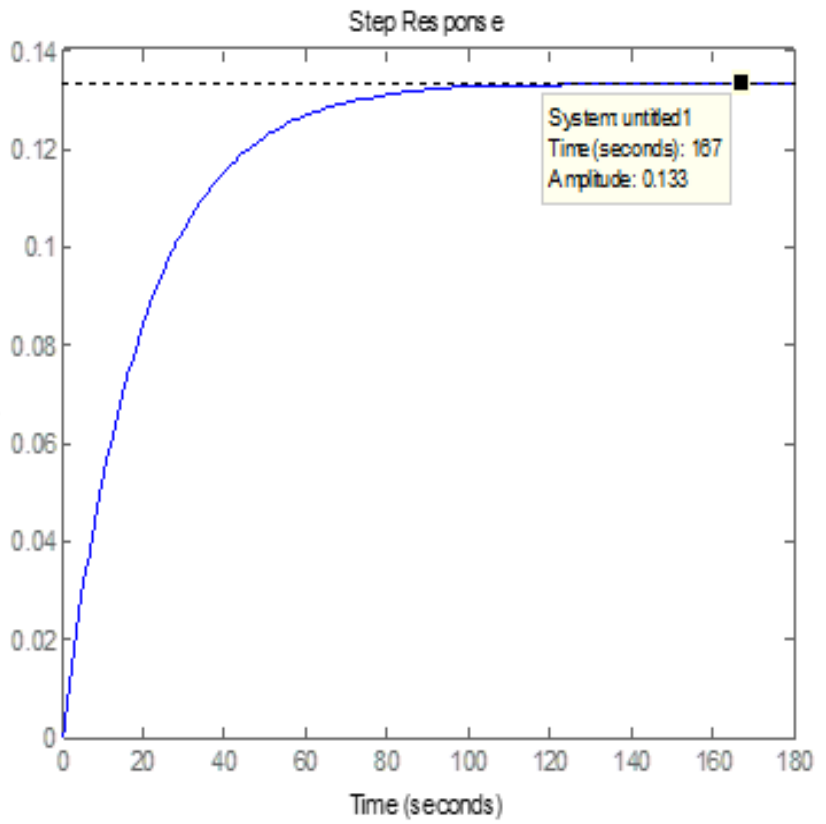

Fig. 10 Step-response of the system having met all the design specifications.

\section{Conclusion}

This work involved the modeling and control design of a cruise control system with set specifications of steady state error less than $1 \%$, overshoot less than $5 \%$ and rise time less than 1.5 seconds achieved using the developed model with the aid of MATLAB. A future work on cruise control system will be to predict future incidence, suggest possible solution to solve the problem, and also, interact with neighborhood vehicles on the highway and automatically adjust itself to the form that would be safe for the passengers, driver of the vehicle and other road users. The use of more sophisticated sensors with higher switching speed semiconductor materials with the knowledge of $\mathrm{AI}$ and machine learning techniques will be required. In fact, such a system having inherent attributes will be suitable to be used as a self-driving robotic vehicle.

\section{References}

[1] Car Bike Tech, (2019). What is cruise control system in cars? Retrieved from: https://carbiketech.com/cruise-control/

[2] RAC (2018) How to use cruise control. Retrieved from: https://www.rac.co.uk/drive/advice/driving-advice/cruisecontrol

[3] ArvindRaj, R., Kumar, S., \& Karthik, S. (2011). Cruise Control Operation from Zero to Preset Speed-Simulation and Implementation. International Journal of Information and Education Technology, 1(1), 9-14. https://doi.org/10.7763/ijiet.2011.v1.2.

[4] Sailan, K., \& Kuhnert, K. D. (2013). Modeling and Design of Cruise Control System with Feedforward for all Terrian Vehicles. 339-349. https://doi.org/10.5121/csit.2013.3828.

[5] Govindaraju, K., Boopathi, S., Ahmed, F. P., Ram, S. T., \& Jagadeeshraja, M. (2015). Embedded Based Vehicle Speed 
Control System Using Wireless Technology. International Journal of Advance Engineering and Research Development, 2(11), 1841-1844. https://doi.org/10.21090/ijaerd.021164.

[6] Diba, F., Arora, A., \& Esmailzadeh, E. (2014). Optimized robust cruise control system for an electric vehicle. Systems Science and Control Engineering, 2(1), 175-182. https://doi.org/10.1080/21642583.2014.891956

[7] Woo, H., Madokoro, H., Sato, K., \& Tamura, Y. (2000). applied sciences Advanced Adaptive Cruise Control Based on Operation Characteristic Estimation and Trajectory Prediction https://doi.org/10.3390/app9224875
[8] Chandramohan, N. A. (2018). Design and Modeling of Adaptive Cruise Control System. University Indianapolis, Indiana.

[9] Khaled, S., \& Klaus, D.K. (2013) Modeling and design of cruise control system with feed forward for all terrain vehicles.

[10] Oguntosin, V., \& Akindele, A. (2019). Design of a joint angle measurement system for the rotary joint of a robotic arm using an Incremental Rotary Encoder. Journal of Physics: Conference Series. 1299(1).

[11] Oguntosin, V., \& Abdulkareem, A. (2020). Design of apneumatic soft actuator controlled via eye tracking and detection, Heliyon. 6(7):e04388, 